\title{
Existence of multiple positive solutions for a truncated Kirchhoff-type system involving weight functions and concave-convex nonlinearities
}

Qingjun Lou ${ }^{*}$ and Yupeng Qin²

"Correspondence:

louqing.jun@163.com

'School of Mathematical Sciences,

University of Jinan, Jinan, P.R. China

Full list of author information is

available at the end of the article

\begin{abstract}
We consider the combined effect of concave-convex nonlinearities on the number of solutions for an indefinite truncated Kirchhoff-type system involving the weight functions. When $\alpha+\beta<4$, since the concave-convex nonlinearities do not satisfy the mountain pass geometry, it is difficult to obtain a bounded Palais-Smale sequence by the usual mountain pass theorem. To overcome the problem, we properly introduce a method of Nehari manifold and then establish the existence of multiple positive solutions when the pair of the parameters is under a certain range.
\end{abstract}

Keywords: Kirchhoff system; Multiple positive solutions; Nehari manifold

\section{Introduction and main results}

In this paper, we consider the existence and multiplicity of positive solutions for the following truncated Kirchhoff-type system involving concave-convex nonlinearities:

$$
\left\{\begin{array}{l}
-M_{1}^{k}\left(\int_{\Omega}|\nabla u|^{2}\right) \Delta u=\lambda f(x)|u|^{q-2} u+\frac{\alpha}{\alpha+\beta}|u|^{\alpha-2} u|v|^{\beta} \quad \text { in } \Omega, \\
-M_{2}^{k}\left(\int_{\Omega}|\nabla v|^{2}\right) \Delta v=\mu g(x)|v|^{q-2} v+\frac{\beta}{\alpha+\beta}|u|^{\alpha}|v|^{\beta-2} v \quad \text { in } \Omega, \\
u, v \in H_{0}^{1}(\Omega),
\end{array}\right.
$$

where $\Omega \subset \mathbb{R}^{N}(N>4)$ is a bounded domain with smooth boundary, $\alpha>1$ and $\beta>1$ satisfy $\alpha+\beta<2^{*}<4$ where $2^{*}:=2 N /(N-2)$ is the critical Sobolev exponent, $1<q<2$, $M_{i}=a_{i}+b_{i} t, a_{i}, b_{i}>0(i=1,2),(\lambda, \mu) \in(0,+\infty) \times(0,+\infty), k<\min \left\{\frac{a_{1}(\alpha+\beta-2)}{2 b_{1}}, \frac{a_{2}(\alpha+\beta-2)}{2 b_{2}}\right\}$,

$$
M_{i}^{k}(s)=\left\{\begin{array}{ll}
M_{i}(s) & \text { if } s \leq k, \\
M_{i}(k) & \text { if } s>k,
\end{array} \quad i=1,2,\right.
$$

and the weight functions $f, g$ satisfy the following conditions:

(F) $f \in C(\bar{\Omega}), f>0$;

(G) $g \in C(\bar{\Omega}), g>0$.

C) The Author(s) 2020. This article is licensed under a Creative Commons Attribution 4.0 International License, which permits use, sharing, adaptation, distribution and reproduction in any medium or format, as long as you give appropriate credit to the original author(s) and the source, provide a link to the Creative Commons licence, and indicate if changes were made. The images or other third party material in this article are included in the article's Creative Commons licence, unless indicated otherwise in a credit line to the material. If material is not included in the article's Creative Commons licence and your intended use is not permitted by statutory regulation or exceeds the permitted use, you will need to obtain permission directly from the copyright holder. To view a copy of this licence, visit http://creativecommons.org/licenses/by/4.0/ 
$\operatorname{Problem}\left(E_{\lambda, \mu, M^{k}}\right)$ is called nonlocal because of the presence of $b_{1} \int_{\Omega}|\nabla u|^{2}$ and $b_{2} \int_{\Omega}|\nabla v|^{2}$, and $b\left(\int_{\Omega}|\nabla u|^{2}\right) \Delta u$ appears in the Kirchhoff equation

$$
\left\{\begin{array}{l}
-\left(a+b \int_{\Omega}|\nabla u|^{2}\right) \Delta u=f(x, u) \quad \text { in } \Omega, \\
u=0 \quad \text { on } \partial \Omega,
\end{array}\right.
$$

related to the stationary analogue of the equation

$$
u_{t t}-\left(a+b \int_{\Omega}|\nabla u|^{2}\right) \Delta u=f(x, u)
$$

where $u$ is the displacement, $f(x, t)$ is the external force, $a$ is the initial tension, and $b$ is related to the intrinsic properties of the string. The equation was first proposed by Kirchhoff [1] as an extension of the classical D'Alembert's wave equation to describe free vibrations of elastic strings. Several existence results for equation $\left(E_{1}\right)$ have been obtained in recent years; see [2-7] and references therein. Moreover, other similar arguments are also obtained; see [8-12].

When $a_{i}=0$ and $b_{i}=1(i=1,2)$, problem $\left(E_{\lambda, \mu, M^{k}}\right)$ becomes

$$
\left\{\begin{array}{l}
-\Delta u=\lambda f(x)|u|^{q-2} u+\frac{\alpha}{\alpha+\beta}|u|^{\alpha-2} u|v|^{\beta} \quad \text { in } \Omega, \\
-\Delta v=\mu g(x)|v|^{q-2} v+\frac{\beta}{\alpha+\beta}|u|^{\alpha}|v|^{\beta-2} v \quad \text { in } \Omega, \\
u=v=0 \text { on } \partial \Omega .
\end{array}\right.
$$

Nowadays scientists and researchers paid more attentions to problem $\left(E_{2}\right)$ with sighchanging weight function. For instance, the case $\alpha+\beta=2^{*}$ is considered in [13], whereas in $[14,15]$ the case $\alpha+\beta<2^{*}$ was studied, and the existence and multiplicity of positive solutions when $(\lambda, \mu)$ belongs to a certain subset of $\mathbb{R}^{2}$ were obtained.

Meanwhile, the problem about Kirchhoff system has been studied. In $[16,17]$ the Kirchhoff system with boundary value shows several physical and biological systems with $u$ and $v$ describing a process depending on the average of itself, such as population densities. Lv and Peng [18] established the existence of positive vector solutions and positive vector ground state solutions by using variational methods and also studied the asymptotic behavior of these solutions. In [19] the authors studied the nonlocal boundary value problem of Kirchhoff-type system, where $\Omega$ is a bounded domain in $\mathbb{R}^{N}, N=1,2,3, \beta \in \mathbb{R}$, $a_{i}, b_{i}, \lambda_{i}>0$ for $i=1,2$, and $p$ and $q$ are two positive numbers satisfying certain conditions. They obtained the existence of positive solutions by the Nehari manifold and mountain pass lemma and the multiplicity by using cohomological index of Fadell and Rabinowitz. Also, they considered the critical case and proved the existence of positive least energy solutions when $\beta$ is sufficiently large.

Inspired by the works mentioned, in this paper, we mainly study the truncated Kirchhofftype system with concave-convex nonlinearities involving $\alpha+\beta<4$, since the case $\alpha+$ $\beta \geq 4$ is trivial, which is easy to be proved by using the method in [20]. To the best of our knowledge, the usual mountain pass theorem cannot be directly applied because the concave-convex nonlinearities do not satisfy the mountain pass geometry, so it is difficult to obtain a bounded Palais-Smale sequence (see Theorem 1.15 in [21]). Hence, in this work, by using the method of Nehari manifold, we overcome this difficulty and obtain the existence of multiple positive solutions. 
Let us state our knowledge framework and main result. For $u \in H_{0}^{1}(\Omega)$, its usual norm is denoted by

$$
\|u\|^{2}=\int_{\Omega}|\nabla u|^{2}
$$

Consider system $\left(E_{\lambda, \mu, M^{k}}\right)$ in the framework of the Sobolev space $H=H_{0}^{1}(\Omega) \times H_{0}^{1}(\Omega)$ with the standard norm

$$
\|(u, v)\|_{H}^{2}=\int_{\Omega}\left(|\nabla u|^{2}+|\nabla v|^{2}\right) .
$$

The energy functional associated with the equation $\left(E_{\lambda, \mu, M^{k}}\right)$ is defined by

$$
\begin{aligned}
I_{\lambda, \mu, M^{k}}(u, v)= & \frac{1}{2} \hat{M}_{1}^{k}\left(\|u\|^{2}\right)+\frac{1}{2} \hat{M}_{2}^{k}\left(\|v\|^{2}\right)-\frac{1}{q} \int_{\Omega}\left(\lambda f|u|^{q}+\mu g|v|^{q}\right) \\
& -\frac{1}{\alpha+\beta} \int_{\Omega}|u|^{\alpha}|v|^{\beta}
\end{aligned}
$$

where $\hat{M}_{i}^{k}(t)=\int_{0}^{t} M_{i}^{k}(s) d s$. It is well known that the functional $I_{\lambda, \mu, M^{k}}$ is of class $C^{1}$. Further, we denote

$$
\Lambda=\left(\left(\lambda\|f\|_{\infty}\right)^{2 /(2-q)}+\left(\mu\|g\|_{\infty}\right)^{2 /(2-q)}\right)^{(2-q) / 2} .
$$

Theorem 1.1 Assume that conditions $(F),(G)$ hold. If $\alpha+\beta<2^{*}$, then there exists $\Lambda_{0}>0$ such that for $0<\Lambda<\Lambda_{0}$, equation $\left(E_{\lambda, \mu, M^{k}}\right)$ has at least two positive solutions $\left(u_{\lambda, \mu, M^{k}}^{+}, v_{\lambda, \mu, M^{k}}^{+}\right)$and $\left(u_{\lambda, \mu, M^{k}}^{-}, v_{\lambda, \mu, M^{k}}^{-}\right)$.

\section{Preliminaries}

Let us introduce the Nehari manifold

$$
N_{\lambda, \mu, M^{k}}=\left\{(u, v) \in H \backslash(0,0) \mid\left\langle I_{\lambda, \mu, M^{k}}^{\prime}(u, v),(u, v)\right\rangle=0\right\},
$$

and denote $\Psi_{\lambda, \mu, M^{k}}(u, v)=\left\langle I_{\lambda, \mu, M^{k}}^{\prime}(u, v),(u, v)\right\rangle$ and $\Phi_{\lambda, \mu, M^{k}}(u, v)=\left\langle\Psi_{\lambda, \mu, M^{k}}^{\prime}(u, v),(u, v)\right\rangle$. If $(u, v) \in N_{\lambda, \mu, M^{k}}$, then

$$
\begin{aligned}
\Phi_{\lambda, \mu, M^{k}}(u, v)= & 2\left(M_{1}^{k}\right)^{\prime}\left(\|u\|^{2}\right)\|u\|^{4}+2\left(M_{2}^{k}\right)^{\prime}\left(\|v\|^{2}\right)\|v\|^{4}+2 M_{1}^{k}\left(\|u\|^{2}\right)\|u\|^{2} \\
& +2 M_{2}^{k}\left(\|v\|^{2}\right)\|v\|^{2}-q \int_{\Omega}\left(\lambda f|u|^{q}+\mu g|v|^{q}\right)-(\alpha+\beta) \int_{\Omega}|u|^{\alpha}|v|^{\beta} \\
= & (2-q) M_{1}^{k}\left(\|u\|^{2}\right)\|u\|^{2}+2\left(M_{1}^{k}\right)^{\prime}\left(\|u\|^{2}\right)\|u\|^{4}+(2-q) M_{2}^{k}\left(\|v\|^{2}\right)\|v\|^{2} \\
& +2\left(M_{2}^{k}\right)^{\prime}\left(\|v\|^{2}\right)\|v\|^{4}-(\alpha+\beta-q) \int_{\Omega}|u|^{\alpha}|v|^{\beta} \\
= & (2-\alpha-\beta) M_{1}^{k}\left(\|u\|^{2}\right)\|u\|^{2}+2\left(M_{1}^{k}\right)^{\prime}\left(\|u\|^{2}\right)\|u\|^{4} \\
& +(2-\alpha-\beta) M_{2}^{k}\left(\|v\|^{2}\right)\|v\|^{2}+2\left(M_{2}^{k}\right)^{\prime}\left(\|v\|^{2}\right)\|v\|^{4} \\
& -(q-\alpha-\beta)\left(\lambda f|u|^{q}+\mu g|v|^{q}\right) .
\end{aligned}
$$


We split $N_{\lambda, \mu, M^{k}}$ into three parts:

$$
\begin{aligned}
& N_{\lambda, \mu, M^{k}}^{+}=\left\{(u, v) \in N_{\lambda, \mu, M^{k}} \mid \Phi_{\lambda, \mu, M^{k}}(u, v)>0\right\}, \\
& N_{\lambda, \mu, M^{k}}^{0}=\left\{(u, v) \in N_{\lambda, \mu, M^{k}} \mid \Phi_{\lambda, \mu, M^{k}}(u, v)=0\right\}, \\
& N_{\lambda, \mu, M^{k}}^{-}=\left\{(u, v) \in N_{\lambda, \mu, M^{k}} \mid \Phi_{\lambda, \mu, M^{k}}(u, v)<0\right\} .
\end{aligned}
$$

The best Sobolev constant $S_{r}\left(1<r<2^{*}\right)$ and $S_{\alpha, \beta}$ are respectively defined by

$$
\begin{aligned}
& S_{r}=\inf _{u \in H_{0}^{1}(\Omega) \backslash\{0\}} \frac{\int_{\Omega}|\nabla u|^{2}}{\left(\int_{\Omega}|u|^{r}\right)^{2 / r}}, \\
& S_{\alpha, \beta}=\inf _{u, v \in H_{0}^{1}(\Omega) \backslash\{0\}} \frac{\int_{\Omega}\left(|\nabla u|^{2}+|\nabla v|^{2}\right)}{\left(\int_{\Omega}|u|^{\alpha}|v|^{\beta}\right)^{2 /(\alpha+\beta)}} .
\end{aligned}
$$

Lemma 2.1 Assume that conditions $(F)$ and $(G)$ hold. Then the energy functional $I_{\lambda, \mu, M^{k}}$ is coercive and bounded below on $N_{\lambda, \mu, M^{k}}$.

Proof For $(u, v) \in N_{\lambda, \mu, M^{k}}$,

$$
\begin{aligned}
& M_{1}^{k}\left(\|u\|^{2}\right)\|u\|^{2}+M_{2}^{k}\left(\|v\|^{2}\right)\|v\|^{2} \\
& \quad=\int_{\Omega}\left(\lambda f|u|^{q}+\mu g|v|^{q}\right)+\int_{\Omega}|u|^{\alpha}|v|^{\beta} .
\end{aligned}
$$

Setting $M_{0}=\min \left\{\frac{a_{1}}{2}-\frac{M_{1}(k)}{\alpha+\beta}, \frac{a_{2}}{2}-\frac{M_{2}(k)}{\alpha+\beta}\right\}$, by the Sobolev and Hölder inequalities we obtain

$$
\begin{aligned}
I_{\lambda, \mu, M^{k}}(u, v)= & I_{\lambda, \mu, M^{k}}(u, v)-\frac{1}{\alpha+\beta} \Psi_{\lambda, \mu, M^{k}}(u, v) \\
= & \left(\frac{1}{2} \hat{M}_{1}^{k}\left(\|u\|^{2}\right)-\frac{1}{\alpha+\beta} M_{1}^{k}\left(\|u\|^{2}\right)\|u\|^{2}\right) \\
& +\left(\frac{1}{2} \hat{M}_{2}^{k}\left(\|v\|^{2}\right)-\frac{1}{\alpha+\beta} M_{2}^{k}\left(\|v\|^{2}\right)\|v\|^{2}\right) \\
& -\frac{\alpha+\beta-q}{(\alpha+\beta) q} \int_{\Omega}\left(\lambda f|u|^{q}+\mu g|v|^{q}\right) .
\end{aligned}
$$

To finish this proof, we need the following claims.

Claim $1 \frac{1}{2} \hat{M}_{1}^{k}\left(\|u\|^{2}\right)-\frac{1}{\alpha+\beta} M_{1}^{k}\left(\|u\|^{2}\right)\|u\|^{2} \geq\left(\frac{a_{1}}{2}-\frac{M_{1}(k)}{\alpha+\beta}\right)\|u\|^{2}$.

Claim $2 \frac{1}{2} \hat{M}_{2}^{k}\left(\|v\|^{2}\right)-\frac{1}{\alpha+\beta} M_{2}^{k}\left(\|v\|^{2}\right)\|v\|^{2} \geq\left(\frac{a_{2}}{2}-\frac{M_{2}(k)}{\alpha+\beta}\right)\|v\|^{2}$.

Claim $3 \int_{\Omega}\left(\lambda f|u|^{q}+\mu g|v|^{q}\right) \leq S_{q}^{-q / 2} \Lambda\|(u, v)\|_{H}^{q}$.

First, by the Sobolev and Hölder inequalities we easily obtain Claim 3. Then, since the proof of Claim 2 is the same as that of Claim 1, here we only give the proof of Claim 1. If 
$\|u\|^{2} \leq k$, then we have that

$$
\begin{gathered}
\frac{1}{2} \hat{M}_{1}^{k}\left(\|u\|^{2}\right)-\frac{1}{\alpha+\beta} M_{1}^{k}\left(\|u\|^{2}\right)\|u\|^{2}-\left(\frac{a_{1}}{2}-\frac{M_{1}(k)}{\alpha+\beta}\right)\|u\|^{2} \\
=\frac{1}{\alpha+\beta} b_{1}\|u\|^{2}\left(k-\|u\|^{2}\right)+\frac{b_{1}}{4}\|u\|^{4} \geq 0,
\end{gathered}
$$

and if $\|u\|^{2}>k$, then we conclude that

$$
\begin{aligned}
& \frac{1}{2} \hat{M}_{1}^{k}\left(\|u\|^{2}\right)-\frac{1}{\alpha+\beta} M_{1}^{k}\left(\|u\|^{2}\right)\|u\|^{2}-\left(\frac{a_{1}}{2}-\frac{M_{1}(k)}{\alpha+\beta}\right)\|u\|^{2} \\
& \quad=\frac{1}{2} \int_{0}^{k} M_{1}(s) d s+\frac{1}{2} \int_{k}^{\|u\|^{2}} M_{1}(k) d s-\frac{a_{1}}{2}\|u\|^{2} \\
& \quad=\frac{1}{4} b_{1} k\left(2\|u\|^{2}-k\right) \geq 0,
\end{aligned}
$$

which completes the proof of Claim 1.

Thus, we could obtain that

$$
\begin{aligned}
I_{\lambda, \mu, M^{k}}(u, v) & \geq\left(\frac{a_{1}}{2}-\frac{M_{1}(k)}{\alpha+\beta}\right)\|u\|^{2}+\left(\frac{a_{2}}{2}-\frac{M_{2}(k)}{\alpha+\beta}\right)\|v\|^{2}-\frac{\alpha+\beta-q}{(\alpha+\beta) q} S_{q}^{-q / 2} \Lambda\|(u, v)\|_{H}^{q} \\
& \geq M_{0}\|(u, v)\|_{H}^{2}-\frac{\alpha+\beta-q}{(\alpha+\beta) q} S_{q}^{-q / 2} \Lambda\|(u, v)\|_{H}^{q} .
\end{aligned}
$$

Since $k<\min \left\{\frac{a_{1}(\alpha+\beta-2)}{2 b_{1}}, \frac{a_{2}(\alpha+\beta-2)}{2 b_{2}}\right\}$, we have $M_{0}>0$. Thus $I_{\lambda, \mu, M^{k}}$ is coercive and bounded below on $N_{\lambda, \mu, M^{k}}$.

Lemma 2.2 Suppose that $\left(u_{0}, v_{0}\right)$ is a local minimizer for $I_{\lambda, \mu, M^{k}}$ on $N_{\lambda, \mu, M^{k}},\left(u_{0}, v_{0}\right) \notin$ $N_{\lambda, \mu, M^{k}}^{0}$. Then $I_{\lambda, \mu, M^{k}}^{\prime}\left(u_{0}, v_{0}\right)=0$ in $H^{-1}$.

Proof We refer to Theorem 2.3 of [22].

Since $2<\alpha+\beta<4$, we have $k<\frac{a_{1}(\alpha+\beta-2)}{2 b_{1}}<\frac{a_{1}(\alpha+\beta-2)}{b_{1}(4-\alpha-\beta)}$ and $k<\frac{a_{2}(\alpha+\beta-2)}{2 b_{2}}<\frac{a_{2}(\alpha+\beta-2)}{b_{2}(4-\alpha-\beta)}$, so that $a_{1}(\alpha+\beta-2)-b_{1}(4-\alpha-\beta) k>0$ and $a_{2}(\alpha+\beta-2)-b_{2}(4-\alpha-\beta) k>0$.

Setting

$$
\tilde{\Lambda}=\frac{\tilde{C}_{2} S_{q}^{q / 2}}{\alpha+\beta-q}\left(\frac{(2-q) \tilde{C}_{1} S_{\alpha, \beta}^{(\alpha+\beta) / 2}}{\alpha+\beta-q}\right)^{(2-q) /(\alpha+\beta-2)},
$$

where $\tilde{C}_{1}=\min \left\{a_{1}, a_{2}, M_{1}(k), M_{2}(k)\right\}$, and $\tilde{C}_{2}=\min \left\{a_{1}(\alpha+\beta-2)-b_{1}(4-\alpha-\beta) k\right.$, $\left.a_{2}(\alpha+\beta-2)-b_{2}(4-\alpha-\beta) k, M_{1}(k)(\alpha+\beta-2), M_{2}(k)(\alpha+\beta-2)\right\}$, we obtain the following result.

Lemma 2.3 Assume that conditions $(F)$ and $(G)$ hold. If $\alpha+\beta<2^{*}$, then $N_{\lambda, \mu, M^{k}}^{0}=\varnothing$ for $\Lambda \in(0, \tilde{\Lambda})$.

Proof For each $(u, v) \in N_{\lambda, \mu, M^{k}}^{0}$, in (2.1), we discuss the problem in four cases. 
Case 1: If $\|u\|^{2} \leq k,\|v\|^{2} \leq k$, and

$$
\begin{aligned}
\Phi_{\lambda, \mu, M^{k}}(u, v)= & (2-q) a_{1}\|u\|^{2}+(4-q) b_{1}\|u\|^{4}+(2-q) a_{2}\|v\|^{2}+(4-q) b_{2}\|v\|^{4} \\
& -(\alpha+\beta-q) \int_{\Omega}|u|^{\alpha}|v|^{\beta}
\end{aligned}
$$

then

$$
\begin{aligned}
(2-q) a\|(u, v)\|_{H}^{2} & \leq(2-q) a_{1}\|u\|^{2}+(2-q) a_{2}\|v\|^{2} \\
& \leq(\alpha+\beta-q) \int_{\Omega}|u|^{\alpha}|v|^{\beta} \\
& \leq(\alpha+\beta-q) S_{\alpha, \beta}^{-(\alpha+\beta) / 2}\|(u, v)\|_{H}^{\alpha+\beta},
\end{aligned}
$$

where $a=\min \left\{a_{1}, a_{2}\right\}>0$.

Case 2: If $\|u\|^{2} \leq k,\|v\|^{2}>k$, and

$$
\begin{aligned}
\Phi_{\lambda, \mu, M^{k}}(u, v)= & (2-q) a_{1}\|u\|^{2}+(4-q) b_{1}\|u\|^{4}+(2-q) M_{2}(k)\|v\|^{2} \\
& -(\alpha+\beta-q) \int_{\Omega}|u|^{\alpha}|v|^{\beta}
\end{aligned}
$$

then

$$
\begin{aligned}
(2-q) a_{1}^{k}\|(u, v)\|_{H}^{2} & \leq(2-q) a_{1}\|u\|^{2}+(2-q) M_{2}(k)\|v\|^{2} \\
& \leq(\alpha+\beta-q) S_{\alpha, \beta}^{-(\alpha+\beta) / 2}\|(u, v)\|_{H}^{\alpha+\beta}
\end{aligned}
$$

where $a_{1}^{k}=\min \left\{a_{1}, M_{2}(k)\right\}$.

Case 3: If $\|u\|^{2}>k,\|v\|^{2} \leq k$, and

$$
\begin{aligned}
\Phi_{\lambda, \mu, M^{k}}(u, v)= & (2-q) M_{1}(k)\|u\|^{2}+(2-q) a_{2}\|v\|^{2}+(4-q) b_{2}\|v\|^{4} \\
& -(\alpha+\beta-q) \int_{\Omega}|u|^{\alpha}|v|^{\beta}
\end{aligned}
$$

then

$$
\begin{aligned}
(2-q) a_{2}^{k}\|(u, v)\|_{H}^{2} & \leq(2-q) M_{1}(k)\|u\|^{2}+(2-q) a_{2}\|v\|^{2} \\
& \leq(\alpha+\beta-q) S_{\alpha, \beta}^{-(\alpha+\beta) / 2}\|(u, v)\|_{H}^{\alpha+\beta}
\end{aligned}
$$

where $a_{2}^{k}=\min \left\{a_{2}, M_{1}(k)\right\}$.

Case 4: If $\|u\|^{2}>k,\|v\|^{2}>k$, and

$$
\Phi_{\lambda, \mu, M^{k}}(u, v)=(2-q) M_{1}(k)\|u\|^{2}+(2-q) M_{2}(k)\|v\|^{2}-(\alpha+\beta-q) \int_{\Omega}|u|^{\alpha}|v|^{\beta}
$$

then

$$
(2-q) M(k)\|(u, v)\|_{H}^{2} \leq(\alpha+\beta-q) \int_{\Omega}|u|^{\alpha}|v|^{\beta}
$$




$$
\leq(\alpha+\beta-q) S_{\alpha, \beta}^{-(\alpha+\beta) / 2}\|(u, v)\|_{H}^{\alpha+\beta}
$$

where $M(k)=\min \left\{M_{1}(k), M_{2}(k)\right\}$.

For (2.2), we also split the proof into four cases as follows.

Case 1: If $\|u\|^{2} \leq k,\|v\|^{2} \leq k$, and

$$
\begin{aligned}
\Phi_{\lambda, \mu, M^{k}}(u, v)= & \left(b_{1}(4-\alpha-\beta)\|u\|^{2}-a_{1}(\alpha+\beta-2)\right)\|u\|^{2} \\
& +\left(b_{2}(4-\alpha-\beta)\|v\|^{2}-a_{2}(\alpha+\beta-2)\right)\|v\|^{2} \\
& +(\alpha+\beta-q) \int_{\Omega}\left(\lambda f|u|^{q}+\mu g|v|^{q}\right),
\end{aligned}
$$

then since $k<\frac{a_{1}(\alpha+\beta-2)}{2 b_{1}}<\frac{a_{1}(\alpha+\beta-2)}{b_{1}(4-\alpha-\beta)}$ and $k<\frac{a_{2}(\alpha+\beta-2)}{2 b_{2}}<\frac{a_{2}(\alpha+\beta-2)}{b_{2}(4-\alpha-\beta)}$, we have $a_{1}(\alpha+\beta-2)-$ $b_{1}(4-\alpha-\beta) k>0$ and $a_{2}(\alpha+\beta-2)-b_{2}(4-\alpha-\beta) k>0$. Thus

$$
\begin{aligned}
& \left(a_{1}(\alpha+\beta-2)-b_{1}(4-\alpha-\beta) k\right)\|u\|^{2}+\left(a_{2}(\alpha+\beta-2)-b_{2}(4-\alpha-\beta) k\right)\|v\|^{2} \\
& \quad \leq(\alpha+\beta-q) \int_{\Omega}\left(\lambda f|u|^{q}+\mu g|v|^{q}\right) \\
& \quad \leq(\alpha+\beta-q) S_{q}^{-q / 2} \Lambda\|(u, v)\|_{H^{q}}^{q}
\end{aligned}
$$

Let $T_{1}=\min \left\{a_{1}(\alpha+\beta-2)-b_{1}(4-\alpha-\beta) k, a_{2}(\alpha+\beta-2)-b_{2}(4-\alpha-\beta) k\right\}$. Then

$$
T_{1}\|(u, v)\|_{H}^{2} \leq(\alpha+\beta-q) S_{q}^{-q / 2} \Lambda\|(u, v)\|_{H}^{q}
$$

Case 2: If $\|u\|^{2} \leq k$ and $\|v\|^{2}>k$, then

$$
\begin{aligned}
\Phi_{\lambda, \mu, M^{k}}(u, v)= & \left(b_{1}(4-\alpha-\beta)\|u\|^{2}-a_{1}(\alpha+\beta-2)\right)\|u\|^{2}-M_{2}(k)(\alpha+\beta-2)\|v\|^{2} \\
& +(\alpha+\beta-q) \int_{\Omega}\left(\lambda f|u|^{q}+\mu g|v|^{q}\right)
\end{aligned}
$$

and

$$
\begin{aligned}
T_{2}\|(u, v)\|_{H}^{2} & \leq\left(a_{1}(\alpha+\beta-2)-b_{1}(4-\alpha-\beta) k\right)\|u\|^{2}+M_{2}(k)(\alpha+\beta-2)\|v\|^{2} \\
& \leq(\alpha+\beta-q) S_{q}^{-q / 2} \Lambda\|(u, v)\|_{H}^{q},
\end{aligned}
$$

where $T_{2}=\min \left\{a_{1}(\alpha+\beta-2)-b_{1}(4-\alpha-\beta) k, M_{2}(k)(\alpha+\beta-2)\right\}$.

Case 3: If $\|u\|^{2}>k,\|v\|^{2} \leq k$, then

$$
\begin{aligned}
\Phi_{\lambda, \mu, M^{k}}(u, v)= & -M_{1}(k)(\alpha+\beta-2)\|u\|^{2}+\left(b_{2}(4-\alpha-\beta)\|v\|^{2}-a_{2}(\alpha+\beta-2)\right)\|v\|^{2} \\
& +(\alpha+\beta-q) \int_{\Omega}\left(\lambda f|u|^{q}+\mu g|v|^{q}\right)
\end{aligned}
$$

and

$$
\begin{aligned}
T_{3}\|(u, v)\|_{H}^{2} & \leq M_{1}(k)(\alpha+\beta-2)\|u\|^{2}+\left(a_{2}(\alpha+\beta-2)-b_{2}(4-\alpha-\beta) k\right)\|v\|^{2} \\
& \leq(\alpha+\beta-q) S_{q}^{-q / 2} \Lambda\|(u, v)\|_{H}^{q},
\end{aligned}
$$


where $T_{3}=\min \left\{M_{1}(k)(\alpha+\beta-2), a_{2}(\alpha+\beta-2)-b_{2}(4-\alpha-\beta) k\right\}$.

Case 4: If $\|u\|^{2}>k,\|v\|^{2}>k$, then

$$
\begin{aligned}
\Phi_{\lambda, \mu, M^{k}}(u, v)= & -M_{1}(k)(\alpha+\beta-2)\|u\|^{2}-M_{2}(k)(\alpha+\beta-2)\|v\|^{2} \\
& +(\alpha+\beta-q) \int_{\Omega}\left(\lambda f|u|^{q}+\mu g|v|^{q}\right)
\end{aligned}
$$

and

$$
\begin{aligned}
T_{4}\|(u, v)\|_{H}^{2} & \leq M_{1}(k)(\alpha+\beta-2)\|u\|^{2}+M_{2}(k)(\alpha+\beta-2)\|v\|^{2} \\
& \leq(\alpha+\beta-q) S_{q}^{-q / 2} \Lambda\|(u, v)\|_{H}^{q}
\end{aligned}
$$

where $T_{4}=\min \left\{M_{1}(k)(\alpha+\beta-2), M_{2}(k)(\alpha+\beta-2)\right\}$.

So, it follows from (2.4)-(2.7) that

$$
(2-q) \tilde{C}_{1}\|(u, v)\|_{H}^{2} \leq(\alpha+\beta-q) S_{\alpha, \beta}^{-(\alpha+\beta) / 2}\|(u, v)\|_{H}^{\alpha+\beta} .
$$

Similarly, by (2.8)-(2.11) we also get that

$$
\tilde{C}_{2}\|(u, v)\|_{H}^{2} \leq(\alpha+\beta-q) S_{q}^{-q / 2} \Lambda\|(u, v)\|_{H}^{q},
$$

and by (2.12)-(2.13) we have

$$
\left(\frac{(2-q) \tilde{C}_{1} S_{\alpha, \beta}^{(\alpha+\beta) / 2}}{\alpha+\beta-q}\right)^{1 /(\alpha+\beta-2)} \leq\|(u, v)\|_{H} \leq\left(\frac{(\alpha+\beta-q) \Lambda}{\tilde{C}_{2} S_{q}^{q / 2}}\right)^{1 /(2-q)} .
$$

Consequently,

$$
\Lambda \geq \frac{\tilde{C}_{2} S_{q}^{q / 2}}{\alpha+\beta-q}\left(\frac{(2-q) \tilde{C}_{1} S_{\alpha, \beta}^{(\alpha+\beta) / 2}}{\alpha+\beta-q}\right)^{(2-q) /(\alpha+\beta-2)}=\tilde{\Lambda} .
$$

Therefore $N_{\lambda, \mu, M^{k}}^{0}=\varnothing$ for $\Lambda \in(0, \tilde{\Lambda})$.

Similarly to the argument of [20], we conclude that for $\Lambda \in(0, \tilde{\Lambda}), N_{\lambda, \mu, M^{k}}=N_{\lambda, \mu, M^{k}}^{+} \cup$ $N_{\lambda, \mu, M^{k}}^{-}$and $N_{\lambda, \mu, M^{k}}^{ \pm} \neq \varnothing$. Denoting

$$
\alpha_{\lambda, \mu, M^{k}}^{+}=\inf _{(u, v) \in N_{\lambda, \mu, M^{k}}^{+}} I_{\lambda, \mu, M^{k}}(u, v), \quad \alpha_{\lambda, \mu, M^{k}}^{-}=\inf _{(u, v) \in N_{\lambda, \mu, M^{k}}^{-}} I_{\lambda, \mu, M^{k}}(u, v),
$$

we have the following conclusion.

Lemma 2.4 Assume that conditions $(F)$ and $(G)$ hold. If $\alpha+\beta<4$, then

(i) $\alpha_{\lambda, \mu, M^{k}}^{+}<0$ for all $\Lambda \in(0, \tilde{\Lambda})$;

(ii) for some $D_{0}>0, \alpha_{\lambda, \mu, M^{k}}^{-}>D_{0}$ for all $\Lambda \in\left(0, \frac{(\alpha+\beta) q M_{0} \tilde{\Lambda}}{\tilde{C}_{2}}\right)$.

In particular, for each $0<\Lambda<\Lambda_{0}=\min \left\{1, \frac{(\alpha+\beta) q M_{0}}{\tilde{C}_{2}}\right\} \tilde{\Lambda}, \alpha_{\lambda, \mu, M^{k}}^{+}=\inf _{(u, v) \in N_{\lambda, \mu, M^{k}}} I_{\lambda, \mu, M^{k}}(u, v)$.

Proof (i) For $(u, v) \in N_{\lambda, \mu, M^{k}}^{+}$, we prove it in four cases. 
Case 1: If $\|u\|^{2} \leq k$ and $\|v\|^{2} \leq k$, then we obtain that

$$
\begin{aligned}
& \left(a_{1}(\alpha+\beta-2)-b_{1}(4-\alpha-\beta) k\right)\|u\|^{2}+\left(a_{2}(\alpha+\beta-2)-b_{2}(4-\alpha-\beta) k\right)\|v\|^{2} \\
& \leq\left(a_{1}(\alpha+\beta-2)-b_{1}(4-\alpha-\beta)\|u\|^{2}\right)\|u\|^{2} \\
& \quad+\left(a_{2}(\alpha+\beta-2)-b_{2}(4-\alpha-\beta)\|v\|^{2}\right)\|v\|^{2} \\
& \quad<(\alpha+\beta-q) \int_{\Omega}\left(\lambda f|u|^{q}+\mu g|v|^{q}\right) .
\end{aligned}
$$

Since $(\alpha+\beta-2) a_{1}-b_{1}(4-\alpha-\beta) k>0$ and $(\alpha+\beta-2) a_{2}-b_{2}(4-\alpha-\beta) k>0$, we have

$$
\begin{aligned}
I_{\lambda, \mu, M^{k}}(u, v)= & \frac{1}{2} \hat{M}_{1}^{k}\left(\|u\|^{2}\right)+\frac{1}{2} \hat{M}_{2}^{k}\left(\|v\|^{2}\right)-\frac{1}{\alpha+\beta} M_{1}^{k}\left(\|u\|^{2}\right)\|u\|^{2} \\
& -\frac{1}{\alpha+\beta} M_{2}^{k}\left(\|v\|^{2}\right)\|v\|^{2}-\frac{\alpha+\beta-q}{(\alpha+\beta) q} \int_{\Omega}\left(\lambda f|u|^{q}+\mu g|v|^{q}\right) \\
= & \frac{\alpha+\beta-2}{2(\alpha+\beta)}\left(a_{1}\|u\|^{2}+a_{2}\|v\|^{2}\right)+\frac{\alpha+\beta-4}{4(\alpha+\beta)}\left(b_{1}\|u\|^{4}+b_{2}\|v\|^{4}\right) \\
& -\frac{\alpha+\beta-q}{q(\alpha+\beta)} \int_{\Omega}\left(\lambda f|u|^{q}+\mu g|v|^{q}\right) \\
< & {\left[\frac{\alpha+\beta-2}{2(\alpha+\beta)} a_{1}+\frac{\alpha+\beta-4}{4(\alpha+\beta)} b_{1} k-\frac{a_{1}(\alpha+\beta-2)-b_{1}(4-\alpha-\beta) k}{q(\alpha+\beta)}\right]\|u\|^{2} } \\
& +\left[\frac{\alpha+\beta-2}{2(\alpha+\beta)} a_{2}+\frac{\alpha+\beta-4}{4(\alpha+\beta)} b_{2} k-\frac{a_{2}(\alpha+\beta-2)-b_{2}(4-\alpha-\beta) k}{q(\alpha+\beta)}\right]\|v\|^{2}
\end{aligned}
$$

$<0$.

Case 2: If $\|u\|^{2} \leq k$ and $\|v\|^{2}>k$, then we get

$$
\begin{aligned}
& \left(a_{1}(\alpha+\beta-2)-b_{1}(4-\alpha-\beta) k\right)\|u\|^{2}+M_{2}(k)(\alpha+\beta-2)\|v\|^{2} \\
& \quad<(\alpha+\beta-q) \int_{\Omega}\left(\lambda f|u|^{q}+\mu g|v|^{q}\right) .
\end{aligned}
$$

Therefore

$$
\begin{aligned}
& I_{\lambda, \mu, M^{k}}(u, v) \\
& =\frac{\alpha+\beta-2}{2(\alpha+\beta)} a_{1}\|u\|^{2}+\frac{\alpha+\beta-4}{4(\alpha+\beta)} b_{1}\|u\|^{4}+\frac{1}{2}\left(\hat{M}_{2}(k)-M_{2}(k) k\right) \\
& \quad+\frac{M_{2}(k)(\alpha+\beta-2)}{2(\alpha+\beta)}\|v\|^{2}-\frac{\alpha+\beta-q}{q(\alpha+\beta)} \int_{\Omega}\left(\lambda f|u|^{q}+\mu g|v|^{q}\right) \\
& <\left(\frac{\alpha+\beta-2}{2(\alpha+\beta)} a_{1}+\frac{\alpha+\beta-4}{4(\alpha+\beta)} b_{1} k-\frac{a_{1}(\alpha+\beta-2)-b_{1}(4-\alpha-\beta) k}{q(\alpha+\beta)}\right)\|u\|^{2} \\
& \quad+\frac{1}{2}\left(a_{2} k+\frac{b_{2} k^{2}}{2}-\left(a_{2}+b_{2} k\right) k\right) \\
& \quad+\frac{M_{2}(k)(\alpha+\beta-2)}{2(\alpha+\beta)}\|v\|^{2}-\frac{M_{2}(k)(\alpha+\beta-2)}{q(\alpha+\beta)}\|v\|^{2}
\end{aligned}
$$


Case 3: If $\|u\|^{2}>k$ and $\|v\|^{2} \leq k$, then we have that

$$
\begin{aligned}
& I_{\lambda, \mu, M^{k}}(u, v) \\
& \quad<\frac{1}{2}\left(a_{1} k+\frac{b_{1} k^{2}}{2}-\left(a_{1}+b_{1} k\right) k\right)+\frac{M_{1}(k)(\alpha+\beta-2)}{2(\alpha+\beta)}\|u\|^{2}-\frac{M_{1}(k)(\alpha+\beta-2)}{q(\alpha+\beta)}\|u\|^{2} \\
& \quad+\left(\frac{\alpha+\beta-2}{2(\alpha+\beta)} a_{2}+\frac{\alpha+\beta-4}{4(\alpha+\beta)} b_{2} k-\frac{a_{2}(\alpha+\beta-2)-b_{2}(4-\alpha-\beta) k}{q(\alpha+\beta)}\right)\|v\|^{2}
\end{aligned}
$$

$<0$.

Case 4: If $\|u\|^{2}>k$ and $\|v\|^{2}>k$, then we deduce that

$$
\begin{aligned}
& I_{\lambda, \mu, M^{k}}(u, v) \\
& \quad<-\frac{b_{1}}{4} k^{2}+\frac{M_{1}(k)(\alpha+\beta-2)(q-2)}{22^{*} q}\|u\|^{2}+\frac{M_{2}(k)(\alpha+\beta-2)(q-2)}{2(\alpha+\beta) q}\|v\|^{2}-\frac{b_{2}}{4} k^{2}
\end{aligned}
$$$$
<0 \text {. }
$$

Therefore $\alpha_{\lambda, \mu, M^{k}}^{+}=\inf _{(u, v) \in N_{\lambda, \mu, M^{k}}^{+}} I_{\lambda, \mu, M^{k}}(u, v)<0$.

(ii) For $(u, v) \in N_{\lambda, \mu, M^{k}}^{-}$, by (2.4)-(2.7) we have

$$
\begin{aligned}
\tilde{C}_{1}(2-q)\|(u, v)\|_{H}^{2} & <(\alpha+\beta-q) \int_{\Omega}|u|^{\alpha}|v|^{\beta} \\
& \leq(\alpha+\beta-q) S_{\alpha, \beta}^{-(\alpha+\beta) / 2}\|(u, v)\|_{H}^{\alpha+\beta},
\end{aligned}
$$

which implies that

$$
\|(u, v)\|_{H}>\left(\frac{\tilde{C}_{1}(2-q) S_{\alpha, \beta}^{(\alpha+\beta) / 2}}{\alpha+\beta-q}\right)^{1 /(\alpha+\beta-2)} .
$$

From (2.3) we get that

$$
\begin{aligned}
I_{\lambda, \mu, M^{k}}(u, v) & \\
\geq & M_{0}\|(u, v)\|_{H}^{2}-\frac{\alpha+\beta-q}{(\alpha+\beta) q} S_{q}^{-q / 2} \Lambda\|(u, v)\|_{H}^{q} \\
> & \left(\frac{\tilde{C}_{1}(2-q) S_{\alpha, \beta}^{(\alpha+\beta) / 2}}{\alpha+\beta-q}\right)^{q /(\alpha+\beta-2)} \\
& \cdot\left(M_{0}\left(\frac{\tilde{C}_{1}(2-q) S_{\alpha, \beta}^{(\alpha+\beta) / 2}}{\alpha+\beta-q}\right)^{(2-q) /(\alpha+\beta-2)}-\frac{\alpha+\beta-q}{(\alpha+\beta) q} S_{q}^{-q / 2} \Lambda\right) .
\end{aligned}
$$

Thus, if $\Lambda<(\alpha+\beta) q M_{0} \frac{\tilde{\Lambda}}{\tilde{C}_{2}}$, then $\alpha_{\lambda, \mu, M^{k}}^{-}>D_{2}$ for some $D_{2}>0$.

\section{The (PS) condition}

Lemma 3.1 Every bounded Palais-Smale sequence for $I_{\lambda, \mu, M^{k}}$ on $H$ has a strongly convergent subsequence. 
Proof Let $\left\{\left(u_{n}, v_{n}\right)\right\}$ be a bounded Palais-Smale sequence for $I_{\lambda, \mu, M^{k}}$ on $H$. Then the sequence $\left\{u_{n}\right\}\left(\left\{v_{n}\right\}\right)$ is bounded on $H_{0}^{1}(\Omega)$. Thus there exist a subsequence $\left\{u_{n}\right\}\left(\left\{v_{n}\right\}\right)$ and $u_{0} \in H_{0}^{1}(\Omega)\left(v_{0} \in H_{0}^{1}(\Omega)\right)$ such that

$$
\begin{array}{ll}
u_{n} \rightarrow u_{0} \text { weakly in } H_{0}^{1}(\Omega) & \left(v_{n} \rightarrow v_{0} \text { weakly in } H_{0}^{1}(\Omega)\right), \\
u_{n} \rightarrow u_{0} \text { strongly in } L^{r}(\Omega) & \left(v_{n} \rightarrow v_{0} \text { strongly in } L^{r}(\Omega)\right) \quad \text { for } 1<r<2^{*} .
\end{array}
$$

Then

$$
\begin{array}{rl}
\left.\left|\int_{\Omega} f\right| u_{n}\right|^{q-2} u_{n}\left(u_{n}-u_{0}\right) d x \mid & \leq\|f\|_{\infty}\left(\int_{\Omega}\left|u_{n}\right|^{q} d x\right)^{(q-1) / q}\left(\int_{\Omega}\left|u_{n}-u_{0}\right|^{q} d x\right)^{1 / q} \\
\rightarrow 0, & \\
\left.\left|\int_{\Omega} g\right| v_{n}\right|^{q-2} v_{n}\left(v_{n}-v_{0}\right) d x \mid \leq & \|g\|_{\infty}\left(\int_{\Omega}\left|v_{n}\right|^{q} d x\right)^{(q-1) / q}\left(\int_{\Omega}\left|v_{n}-v_{0}\right|^{q} d x\right)^{1 / q} \\
\rightarrow 0, & \cdot\left(\int_{\Omega}\left|u_{n}-u_{0}\right|^{\alpha+\beta} d x\right)^{1 /(\alpha+\beta)} \\
\left.\left|\int_{\Omega}\right| u_{n}\right|^{\alpha-2} u_{n}\left(u_{n}-u_{0}\right)\left|v_{n}\right|^{\beta} d x \mid \leq & \left(\int_{\Omega}\left|u_{n}\right|^{\alpha+\beta} d x\right)^{(\alpha-1) /(\alpha+\beta)} \\
& \left(\int_{\Omega}\left|v_{n}\right|^{\alpha+\beta} d x\right)^{\beta /(\alpha+\beta)} \\
\rightarrow 0 & 0,
\end{array}
$$

and

$$
\begin{aligned}
\left.\left|\int_{\Omega}\right| u_{n}\right|^{\alpha}\left|v_{n}\right|^{\beta-2} v_{n}\left(v_{n}-v_{0}\right) d x \mid \leq & \left(\int_{\Omega}\left|u_{n}\right|^{\alpha+\beta} d x\right)^{\alpha /(\alpha+\beta)}\left(\int_{\Omega}\left|v_{n}-v_{0}\right|^{\alpha+\beta} d x\right)^{1 /(\alpha+\beta)} \\
& \cdot\left(\int_{\Omega}\left|v_{n}\right|^{\alpha+\beta} d x\right)^{(\beta-1) /(\alpha+\beta)} \\
\rightarrow & 0
\end{aligned}
$$

as $n \rightarrow \infty$. Since $\left\{\left(u_{n}, v_{n}\right)\right\}$ is a Palais-Smale sequence for $I_{\lambda, \mu, M^{k}}$, it follows that

$$
\begin{aligned}
& \left\langle I_{\lambda, \mu, M^{k}}^{\prime}\left(u_{n}, v_{n}\right),\left(u_{n}-u_{0}, 0\right)\right\rangle \\
& =M_{1}^{k}\left(\left\|u_{n}\right\|^{2}\right) \int_{\Omega} \nabla u_{n} \nabla\left(u_{n}-u_{0}\right) d x-\lambda \int_{\Omega} f\left|u_{n}\right|^{q-2} u_{n}\left(u_{n}-u_{0}\right) d x \\
& \quad-\frac{\alpha}{4} \int_{\Omega}\left|u_{n}\right|^{\alpha-2} u_{n}\left(u_{n}-u_{0}\right)\left|v_{n}\right|^{\beta} d x \\
& \quad \rightarrow 0
\end{aligned}
$$

and

$$
\left\langle I_{\lambda, \mu, M^{k}}^{\prime}\left(u_{n}, v_{n}\right),\left(0, v_{n}-v_{0}\right)\right\rangle
$$




$$
\begin{aligned}
= & M_{2}^{k}\left(\left\|v_{n}\right\|^{2}\right) \int_{\Omega} \nabla v_{n} \nabla\left(v_{n}-v_{0}\right) d x-\mu \int_{\Omega} g\left|v_{n}\right|^{q-2} v_{n}\left(v_{n}-v_{0}\right) d x \\
& -\frac{\beta}{4} \int_{\Omega}\left|u_{n}\right|^{\alpha}\left|v_{n}\right|^{\beta-2} v_{n}\left(v_{n}-v_{0}\right) d x \\
\rightarrow & 0
\end{aligned}
$$

as $n \rightarrow \infty$. By (3.1), (3.3), and (3.5) we get that

$$
\int_{\Omega} \nabla u_{n} \nabla\left(u_{n}-u_{0}\right) d x \rightarrow 0 \quad \text { as } n \rightarrow \infty
$$

By (3.2), (3.4), and (3.6) we obtain that

$$
\int_{\Omega} \nabla v_{n} \nabla\left(v_{n}-v_{0}\right) d x \rightarrow 0 \quad \text { as } n \rightarrow \infty .
$$

Thus

$$
\left\|\left(u_{n}, v_{n}\right)-\left(u_{0}, v_{0}\right)\right\|_{H}^{2}=\left\|u_{n}-u_{0}\right\|^{2}+\left\|v_{n}-v_{0}\right\|^{2} \rightarrow 0 \quad \text { as } n \rightarrow \infty
$$

Proof of Theorem 1.1 Take $\Lambda<\Lambda_{0}$. By Lemma 2.1 and the Ekeland variational principle [23] there exist two bounded minimizing sequences $\left\{\left(u_{n}^{ \pm}, v_{n}^{ \pm}\right)\right\}$for $I_{\lambda, \mu, M^{k}}$ on $N_{\lambda, \mu, M^{k}}^{ \pm}$such that

$$
I_{\lambda, \mu, M^{k}}\left(u_{n}^{ \pm}, v_{n}^{ \pm}\right)=\alpha_{\lambda, \mu, M^{k}}^{ \pm}+o(1), \quad I_{\lambda, \mu, M^{k}}^{\prime}\left(u_{n}^{ \pm}, v_{n}^{ \pm}\right)=o(1) \quad \text { on } H^{-1}
$$

By Lemma 3.1 there exist subsequences $\left\{\left(u_{n}^{ \pm}, v_{n}^{ \pm}\right)\right\}$and $\left(u_{\lambda, \mu, M^{k}}^{ \pm}, v_{\lambda, \mu, M^{k}}^{ \pm}\right) \in H$, the nonzero solutions of the equation $\left(E_{\lambda, \mu, M^{k}}\right)$, such that $\left(u_{n}^{ \pm}, v_{n}^{ \pm}\right) \rightarrow\left(u_{\lambda, \mu, M^{k}}^{ \pm}, v_{\lambda, \mu, M^{k}}^{ \pm}\right)$strongly in $H$. So $\left(u_{\lambda, \mu, M^{k}}^{ \pm}, v_{\lambda, \mu, M^{k}}^{ \pm}\right) \in N_{\lambda, \mu, M^{k}}^{ \pm}$and $I_{\lambda, \mu, M^{k}}\left(u_{\lambda, \mu, M^{k}}^{ \pm}, v_{\lambda, \mu, M^{k}}^{ \pm}\right)=\alpha_{\lambda, \mu, M^{k}}^{ \pm}$. Since $I_{\lambda, \mu, M^{k}}\left(u_{\lambda, \mu, M^{k}}^{ \pm}, v_{\lambda, \mu, M^{k}}^{ \pm}\right)=I_{\lambda, \mu, M^{k}}\left(\left|u_{\lambda, \mu, M^{k}}^{ \pm}\right|,\left|v_{\lambda, \mu, M^{k}}^{ \pm}\right|\right)$, by Lemma 2.2 and Lemma 2.4 we could obtain that $\left(u_{\lambda, \mu, M^{k}}^{+}, v_{\lambda, \mu, M^{k}}^{+}\right)$and $\left(u_{\lambda, \mu, M^{k}}^{-}, v_{\lambda, \mu, M^{k}}^{-}\right)$are two distinct solutions of equation $\left(E_{\lambda, \mu, M^{k}}\right)$ such that $u_{\lambda, \mu, M^{k}}^{ \pm} \geq 0$ and $v_{\lambda, \mu, M^{k}}^{ \pm} \geq 0$ in $\Omega$. By an argument similar to Lemma 2.6 and Theorem 3.2 in [15] we get $u_{\lambda, \mu, M^{k}}^{ \pm} \neq 0$ and $v_{\lambda, \mu, M^{k}}^{ \pm} \neq 0$. By the strong maximum principle [24] we get that $u_{\lambda, \mu, M^{k}}^{ \pm}>0$ and $v_{\lambda, \mu, M^{k}}^{ \pm}>0$.

\section{Acknowledgements}

The authors sincerely thank the reviewers for their valuable suggestions and useful comments.

Funding

This work is supported by the Key Scientific Research Projects of the Higher Education Institutions of Henan Province (20B410001) and the Doctoral Fund of Henan Institute of Technology (KQ1860).

\section{Availability of data and materials}

All data generated or analyzed during this study are included in this paper.

\section{Competing interests}

The authors declare that they have no competing interests.

\section{Authors' contributions}

The authors declare that the study was realized in collaboration with the same responsibility. Both authors read and approved the final manuscript.

\section{Author details}

${ }^{1}$ School of Mathematical Sciences, University of Jinan, Jinan, P.R. China. ${ }^{2}$ School of Science, Henan Institute of

Technology, Xinxiang, P.R. China. 


\section{Publisher's Note}

Springer Nature remains neutral with regard to jurisdictional claims in published maps and institutional affiliations.

Received: 26 April 2019 Accepted: 18 February 2020 Published online: 27 February 2020

\section{References}

1. Kirchhoff, G.: Mechanik. Teubner, Leipzig (1883)

2. He, X., Zou, W.: Infinitely many positive solutions for Kirchhoff-type problems. Nonlinear Anal. 70, 1407-1414 (2009)

3. He, X., Zou, W.: Multiplicity of solutions for a class of Kirchhoff type problems. Acta Math. Appl. Sin. 26, 387-394 (2010)

4. He, X., Zou, W.: Existence and concentration behavior of positive solutions for a Kirchhoff equation in $\mathbb{R}^{3}$. J. Differ. Equ. $252,1813-1834(2012)$

5. Ma, T., Munoz Rivera, J.: Positive solutions for a nonlinear nonlocal elliptic transmission problem. Appl. Math. Lett. 16, 243-248 (2003)

6. Perera, K., Zhang, Z.: Nontrivial solutions of Kirchhoff-type problems via the Yang index. J. Differ. Equ. 221, 246-255 (2006)

7. Lou, Q: A Kirchhoff-type equation involving critical exponent and sign-changing weight functions in dimension four. Math. Methods Appl. Sci. 41, 5159-5177 (2018)

8. Tariboon, J., Ntouyas, S., Agarwal, P.: New concepts of fractional quantum calculus and applications to impulsive fractional q-difference equations. Adv. Differ. Equ. 2015, Article ID 18 (2015)

9. Ruzhansky, M., et al. (eds.): Advances in Real and Complex Analysis with Applications. Springer, Singapore (2018)

10. Ruzhansky, M., et al. (eds.): Advances in Real and Complex Analysis with Applications. (2017)

11. Liu, X., Zhang, L., Agarwal, P., Wang, G.: On some new integral inequalities of Gronwall-Bellman-Bihari type with delay for discontinuous functions and their applications. Indag. Math. 27, 1-10 (2016)

12. Agarwal, P., El-Sayedc, A.: Non-standard finite difference and Chebyshev collocation methods for solving fractional diffusion equation. Phys. A, Stat. Mech. Appl. 500, 40-49 (2017)

13. Chen, C., Wu, T.: The Nehari manifold for indefinite semilinear elliptic systems involving critical exponent. Appl. Math. Comput. 218, 10817-10828 (2012)

14. Brown, K., Wu, T.: A semilinear elliptic system involving nonlinear boundary condition and sign-changing weight function. J. Math. Anal. Appl. 337, 1326-1336 (2008)

15. $\mathrm{Wu}, \mathrm{T}$ :: The Nehari manifold for a semilinear elliptic system involving sign-changing weight functions. Nonlinear Anal. $68,1733-1745$ (2008)

16. Afrouzi, G., Mirzapour, M., Rădulescu, V.: Nonlocal fourth-order Kirchhoff systems with variable growth: low and high energy solutions. Collect. Math. 67, 207-223 (2016)

17. Afrouzi, G., Zahmatkesh, H., Shakeri, S.: Existence results for a class of Kirchhoff type systems with Caffarelli-Kohn-Nirenberg exponents. An. Ştiinţ. Univ. 'Ovidius' Constanţa, Ser. Mat. 24, 83-94 (2016)

18. Lv, D., Peng, S.: Existence and asymptotic behavior of vector solutions for coupled nonlinear Kirchhoff-type systems. J. Differ. Equ. 263, 8947-8978 (2017)

19. Zhang, Z., Sun, Y.: Existence and multiplicity of solutions for nonlocal systems with Kirchhoff type. Acta Math. Appl. Sin. Engl. Ser. 32, 35-54 (2016)

20. Chen, C., Kuo, Y., Wu, T.: The Nehari manifold for a Kirchhoff type problem involving sign-changing weight functions. J. Differ. Equ. 250, 1876-1908 (2011)

21. Willem, M.: Minimax Theorems. Birkhäuser Boston, Boston (1996)

22. Brown, K., Zhang, Y.: The Nehari manifold for a semilinear elliptic equation with a sign-changing weight function. J. Differ. Equ. 193, 481-499 (2003)

23. Ekeland, I.: On the variational principle. J. Math. Anal. Appl. 47, 324-353 (1974)

24. Trudinger, N.: On Harnack type inequalities and their application to quasilinear elliptic equations. Commun. Pure Appl. Math. 20, 721-747 (1967)

\section{Submit your manuscript to a SpringerOpen ${ }^{\circ}$ journal and benefit from:}

- Convenient online submission

- Rigorous peer review

- Open access: articles freely available online

- High visibility within the field

- Retaining the copyright to your article

Submit your next manuscript at $\gg$ springeropen.com 